\title{
Corneal Intrastromal Implantation Surgery by means of MyoRing Corneal Implant for the Treatment of Keratoconus: A Review
}

\author{
${ }^{1}$ Matthias Macsek, ${ }^{2}$ Johannes Steinberg, ${ }^{3}$ Stephan Linke, ${ }^{4}$ Francisco Arnalich, ${ }^{5}$ Marco Alberti, ${ }^{6}$ Albert Daxer
}

\begin{abstract}
This review provides information about MyoRing treatment of keratoconus (KC). MyoRing is a complete ring implant that is inserted into the cornea via a narrow lamellar entrance between two corneal layers at $300 \mu \mathrm{m}$ depth beneath the corneal surface. Since MyoRing is a complete ring it can achieve both visual rehabilitation and stop of progression. The procedure is very safe and effective and lasts only 10 to 15 minutes. It is performed under topical anesthesia and is intra- as well as postoperatively free of pain. Complications are extremely rare.
\end{abstract}

Keywords: Cornea, Intracorneal ring, Keratoconus, MyoRing.

How to cite this article: Macsek M, Steinberg J, Linke S, Arnalich F, Alberti M, Daxer A. Corneal Intrastromal Implantation Surgery by means of MyoRing Corneal Implant for the Treatment of Keratoconus: A Review. Int J Kerat Ect Cor Dis 2018;7(1):50-60.

\section{Source of support: Nil}

Conflict of interest: None

\section{KERATOCONUS}

Keratoconus is a progressive, noninflammatory disease of the cornea characterized by vision loss, irregular corneal shape, and corneal thinning. ${ }^{1}$

\section{Symptoms, Diagnosis, and Epidemiology}

\section{Symptoms}

In the early clinical stages, irregular deformations of the corneal surface lead to slightly blurred vision and

${ }^{1}$ Department of Ophthalmology, St. Pölten University Hospital St. Pölten, Austria

2,3Department of Ophthalmology, Medical University Hamburg Hamburg, Germany

${ }^{4}$ Department of Ophthalmology, Hospital Ramon y Cajal, Madrid Spain

${ }^{5}$ Department of Ophthalmology, Centro Medico Italiano, Milan Italy

${ }^{6}$ International Keratotonus Center, Wels, Austria; Department of Ophthalmology, Medical University of Innsbruck, Innsbruck Austria

Corresponding Author: Albert Daxer, International Keratoconus Center, Wels, Austria; Department of Ophthalmology, Medical University of Innsbruck, Innsbruck, Austria, e-mail: daxer@ gutsehen.at sometimes increased sensitivity to bright lights. When progressing, vision may become more and more distorted due to increasing myopia and higher order aberrations including irregular astigmatism. Because of the inter-eye asymmetry in $\mathrm{KC}$, usually one eye demonstrates noticeably worse symptoms.

Frequently occurring symptoms in clinical manifest KC (i.e., KC stages including morphological changes of the corneal surface/topography) are:

- Distorted vision at all distances

- Noticeably worse vision in one eye

- Double vision in one eye

- "Ghost" images-the appearance of several images when looking at one object

- Shadows-typically like a comet tail (also known as "vertical coma")

- Poor night vision

- Increased light sensitivity

\section{Diagnosis}

Defined as a progressive, bilateral ectasia of the cornea, the diagnosis of $\mathrm{KC}$ is originally based on changing refraction and morphological signs diagnosed with the slit lamp (iron deposition in the epithelial basement membrane "Fleischer rings"), breaks in Bowman's layer (Vogt's striae) and focal corneal thinning. ${ }^{1}$

The development of specialized diagnostic devices focusing on the corneal curvature (especially the PlacidoTopography in 1880) helped to identify this ectatic disease in earlier stages (i.e., before gross morphological changes at the slit lamp occur). ${ }^{2}$ Retrospectively, especially two independent developments gave rise to a growing interest and further improvement of $\mathrm{KC}$ diagnostics. The increased numbers of corneal-refractive procedures after the introduction of excimer laser vision correction in the late 1980s and corneal cross-linking (CXL), as the first sufficient therapy to stop the progression of $\mathrm{KC}$, in the late 1990s. ${ }^{3}$ Although screening for advanced KC with corneal topography is a straightforward procedure, screening eyes with subclinical KC (scKC) (i.e., early stages without clinically relevant topographic characteristics) remains challenging. ${ }^{4}$ However, because even subtle topographical changes potentially lead to a decline of 
the visual acuity, further strategies have been adopted to identify KC especially on a subtopographical level. Unfortunately, because of the absence of an objective, device-independent and up-do-date classification, the definition of "normal," scKC, and early $\mathrm{KC}$ is not coherent in current studies, leading to a distinct bias of the reported results. ${ }^{5}$

A standard method in current scKC screening is the corneal tomography: Using scanning slit-based devices- $\left(\right.$ Orbscan $\left.^{\circledR}\right)$, Scheimpflug, or optical coherence tomography (OCT) - techniques, different devices enable a high-resolution examination and analysis of the entire corneal morphology. ${ }^{6}$ Concentrating on current, highpublished studies, especially point-by-point corneal thickness (progression) analyses and the analysis of posterior elevation pattern reaches an area under the receiver operating characteristic curve (AUROC) of up to 0.91 for scKC screening. ${ }^{7}$ Another study, concentrating on epithelial changes in scKC by using OCT could demonstrate an AUROC of up to $0.985 .{ }^{8}$

A different diagnostic approach is wavefront analyses: With this technique, point-by-point corneal curvature analyses of the anterior and posterior corneal surface are utilized to calculate mathematical wavefront patterns, subdivided and analyzed as Zernike polynomes. Thereby, Saad and Gatinel ${ }^{9}$ and Bühren et $\mathrm{al}^{10}$ demonstrated an AUROC of 0.985 (Saad) and 0.956 (Bühren) to distinguish between normal and scKC eyes with topographically only minor irregularities. Saad and Gatinel ${ }^{9}$ and Bühren et $\mathrm{al}^{10}$ used different definitions of scKC. Whereas Saad and Gatinel ${ }^{9}$ defined both groups using the automated corneal classification software of a corneal topographic analyzing system (OPD-Scan, NIDEK Co. Ltd., Gamagori, Japan), Bühren et $\mathrm{al}^{10}$ defined their scKC by an inferiorsuperior value of $<1.4$ and other topographic patterns, and their NE by retrospectively analyzed Orbscan analyses of patients with an uneventful post-LASIK follow-up of 12 months or more.

The latest approach to improve scKC screening is in vivo biomechanical analyses. The aim of this methodology is to identify KC even before changes of the corneal morphology occur. With the ocular response analyzer (ORA ${ }^{\circledR}$, Reichert) and the corneal visualization Scheimpflug technology (CST ${ }^{\circledR}$, Oculus), two different devices have been developed trying to detect an irregular kinetic response of the cornea to an applied air-puff-impulse (CST: Scheimpflug technology, highspeed camera/single air-impulse; ORA: Infrared signal peaks/pulsed jet of air turned off after the applanation of the cornea). ${ }^{11}$

Whereas the original ORA analyzed mainly two parameters (corneal hysteresis and corneal resistance factor), the current version (2.0) analyzes the deforma- tion dynamics of the cornea, such as height, slope, and width, using 37 "wavefront" parameters. A current study by Luz et $\mathrm{al}^{12}$ reports an AUROC of up to $0.721 \pm 0.065$ to identify (topographically normal) KC eyes with the ORA-wavefront parameters.

The latest CST version offers a Corvis biomechanical index' (CBI) as the most comprehensive index. Currently, there are no studies available analyzing scKC vs normal eyes. ${ }^{13}$ However, studies are in progress testing the capability of the CBI alone and in combination with Pentacam indices generating a combined topographical and biomechanical index to improve scKC screening. Recent combinations of the ORA and tomographical analyses demonstrated an AUROC of up to 0.95.

In conclusion, the corneal topography and tomography represent the gold standard in $\mathrm{KC}$ screening and other techniques should be used as second line/complementary device. However, especially the combination of biomechanical analyses with advanced topography/ tomography devices seem to have the ability to further improve early (i.e., subclinical/subtopographical) KC screening.

\section{Epidemiology}

Stated by Woodward et al, "14 "the most frequently cited occurrence of $\mathrm{KC}$ is $1: 2,000$. This value is based on a registration study in the United States that was conducted from 1935 until 1982. This study reported a prevalence of 54.5 cases per 100,000 individuals." 15

Reviewing epidemiological data on $\mathrm{KC}$, two things should be kept in mind: The time when the data were collected (because the improvement of the diagnostic devices) and the region the data originated/ethnic heterogeneity of the population (because of different cultural influences regarding the intrafamily sexual reproduction).

An example for the diversity of current data are two register studies from European countries: A nationwide registration study to the age-specific incidence and prevalence of KC conducted in the Netherlands based on data from an insurance company covering 30\% (>4.3 million) of the residents reported an annual incidence of $\mathrm{KC}$ of 1:7,500 in persons between 10 and 40 years of age with an estimated prevalence in the general population of 1:375. The mean age at the diagnosis was 28 years and the male/female-ratio was $6 / 4 .^{16}$

A Danish registry study from 2007 analyzing the entire population based on their entry in the National Patient Registry revealed an incidence of $1.3 / 100,000$ and a prevalence of $87 / 100,000 .^{17}$

The published world-wide prevalence ranges from $1 / 500,000$ (Urals, Russia) $^{18}$ to $2.3 \%$ (Central India, 
combined with a high prevalence of consanguinity $)^{19}$ and $3.2 \%$ (Israel) ${ }^{20}$

A study analyzing sociodemographic factors in $\mathrm{KC}$ patients including over 16,000 records from a nationwide United States managed care network using multivariable regression modeling to account for confounding factors could demonstrate a higher odds ratio of KC in Black (57\%) and Latinos (43\%) compared with Whites, whereas Asian Americans presented a 39\% lower odds than Whites. ${ }^{14}$ In contrast to the American study including Asian Americans, two studies analyzing the UK population revealed a higher odds ratio for Asians than Whites [4.4 and 7.5 higher odds for (mostly Muslim) Indian, Pakistani, and Bangladeshi]. ${ }^{21,22}$

Common findings in epidemiological studies include a higher risk of male $v$ s female (about 6:4), of patients with sleep apnea, atopic disease/asthma, or Down syndrome (up to 6:1). For diabetes mellitus, a decreasing risk of 20 to $50 \%$, depending on the severity of the disease, could be identified. ${ }^{14,23}$

Next to all these epidemiological data it is important to understand that, because of the young onset of this chronic disease (mean age at diagnosis equals approximately 28 years ${ }^{16,24}$ ), even modest visual deficits often lead to a disproportionate impact on quality of life and the economic burden of the treatment of KC represents a significant public health concern. ${ }^{25,26}$

\section{Ultrastructure and Biomechanics of the Cornea}

The cornea consists roughly of five principal components: Epithelium, Bowman's membrane, stroma, Descemet membrane (DM), and endothelium. The stroma represents some $90 \%$ of the corneal thickness and is commonly understood as the "corneal tissue". This unique connective tissue is avascular, transparent, and has an immune privilege. ${ }^{27}$ It is responsible for the transparency and the regular shape of the cornea and therefore, for the optical properties of the eye, as well as for the refractive state of the eye (in connection with the axial length) and for the biomechanics of the cornea. The corneal stroma is stacked in some 200 consecutive lamellae of type I collagen fibrils. ${ }^{28}$ Within each lamella, the collagen fibrils run parallel to each other and shows short-range ordered arrangement, which is responsible for the corneal transparency. ${ }^{29}$ The orientation and arrangement of the successively stacked collagen lamellae throughout the entire stroma maintains the biomechanics of the tissue and constitutes the shape of the cornea and the optical function of the eye. ${ }^{30}$ When approaching the periphery of the cornea, the collagen fibrils turn into a circular path forming the limbus. ${ }^{31}$ These ultrastructural properties result in a much higher stiffness at the limbus compared with the cornea and in the spherical dome model of the cornea, which considers the cornea to be mounted at the limbus. ${ }^{32}$ This knowledge is helpful in calculating the effect of corneal implants on the biomechanics of the cornea. $^{33}$

\section{TREATMENT METHODS}

\section{Contact Lens}

The first description of using contact lenses (CLs) to correct the optics in $\mathrm{KC}$ is found from the physician and physiologist Fick. ${ }^{34}$

Nowadays CL fitting is an essential part of providing the appropriate optical rehabilitation in different stages of $\mathrm{KC}$ and can be a great improvement of patients' quality of life. ${ }^{35}$ However, it remains a complex and challenging process. Whereas patients in the early stages of $\mathrm{KC}$ might be sufficiently treated with spectacles, CLs are the mainstay of nonsurgical visual correction in advanced $\mathrm{KC} .{ }^{36}$ Although the variety of available CL has greatly expanded ${ }^{37}$ rigid gas-permeable (RGP) CLs still are the mainstay and most common treatment option especially in early to moderate stages of KC. ${ }^{38}$ The mode of action for RGP CL is masking corneal irregularities, reducing higher order aberrations and thereby improving visual acuity. The selection of the proper CL has to be based on various parameters including material and design. The goal is to achieve a smooth interaction with the keratoconic cornea. ${ }^{39}$

Previous studies have already brought up the concern that a prolonged use of CL alters the underlying corneal cell morphology in healthy as well as in keratoconic corneas. ${ }^{40}$ Mechanical injury to the corneal epithelium caused by $\mathrm{CL}$ on a keratoconic cornea is believed to result in the release of proapoptotic cytokines comparable to the process demonstrated after $\mathrm{CXL}^{41}$ or photorefractive keratectomy. ${ }^{42}$ Pre-CL fitting assessment of the individual corneal shape and evaluation of the transparency of the cornea are essential. The analysis of the corneal shape has greatly improved over the last decades. Placido- and Scheimpflug imaging have become the gold standard techniques to diagnose $\mathrm{KC}$, monitor progression, and optimize CL fitting. ${ }^{5}$ Recently new emerging technologies beyond Scheimpflug-corneal tomography, such as anterior segment OCT provide additional in vivo microstructural information for the clinician and CL fitter. ${ }^{36}$

The CL modalities for KC include soft and rigid design and material: The major limitation of traditional soft CL is the reduced ability to mask irregular astigmatism. Given these circumstances, the use of traditional soft CL is limited to scKC and early stages of KC. ${ }^{43}$ Recently, 
custom-made, aberration-controlled soft CL designs have been developed. ${ }^{44}$ Rigid CLs maintain the architectural structure on the eye. Therefore, they create a thin lacrimal lens between the irregular anterior corneal surface and the posterior lens surface. This results in reducing the astigmatic error but does not necessarily normalize higher-order aberrations. ${ }^{45}$

As published by Downie and Lindsay ${ }^{36}$ in their comprehensive review on CL management of $\mathrm{KC}$, after a period of focusing on rigid corneal lenses, the previous 20 to 30 years are characterized by CL practice paying more and more attention to rigid lens modalities with larger diameter, such as corneo-scleral and miniscleral lenses. These devices bear the advantage of enhanced on-eye stability and decreased lens awareness. Consistent effort has been paid to optimize the design of rigid CL used for the treatment of KC. On the one hand, a steeper back optic zone radius (BOZR) is supposed to fit the conical nature of the central cornea and to reduce the touch at the corneal apex. On the contrary, peripheral curves with progressively flatter radii are supposed to clear the peripheral cornea. The most common design of rigid CL has been the multicurve lens, which consists of multiple spherical radii. ${ }^{36}$

This design bears the advantage of readily changeable parameters including the BOZR, total lens diameter, back peripheral curve radii, back peripheral curve widths, and back optic zone diameter. ${ }^{46}$ In addition, due to improvements in manufacturing and technology, aspheric lenses are also available for the optical correction in patients with $\mathrm{KC} .{ }^{36} \mathrm{In} \mathrm{KC}$ a significant asymmetry in the corneal contour is usually found ${ }^{5}$; therefore, quadrant-specific lens designs, with different edge lifts incorporated into each quadrant of the lens are another innovation in rigid lens designs. As mentioned before, CL fitting remains a demanding task. Comorbidities, such as atopic diseases, including eczema, asthma, and allergic rhino conjunctivitis, can present major hurdles to successful CL wear and should therefore, be controlled by timely and appropriate ophthalmic care. ${ }^{36}$ Innovation in imaging technologies, such as corneal topography, OCT, and in vivo confocal microscopy, has improved $\mathrm{CL}$ fitting. In conclusion, contemporary advances in diagnostics, CL designs, and materials are continuously expanding the range of therapeutic options in patients with corneal irregularity.

\section{Cross-linking}

Introduced in 1998, CXL represented the first therapy for $\mathrm{KC}$, offering a biomechanical strengthening of the otherwise ectatic and thereby destabilizing cornea. ${ }^{3,47}$ Despite the absence of a global consensus on when to use CXL in KC, the most consistent criterion in the literature is an increase of the maximum keratometry (Kmax) of $\geq 1$ D. Further (inhomogeneous) criteria include an increase of the spherical equivalent or astigmatism and decrease of visual acuity. ${ }^{48}$ The principle of CXL is to create oxygen free radicals, which initiate the cross-linking by bridging (mainly) amino groups between corneal collagen fibers. To start this biochemical process, a photosensitizer (riboflavin, vitamin $B_{2}$ ) is applied within the corneal stroma and is then activated by the irradiation with an ultraviolet-A (UV-A; $370 \mathrm{~nm})$ source. Therefore, from a biochemical perspective, the CXL represents an intrastromal photopolymerization process.

The first CXL procedure was named "Dresden protocol" because of its introduction at the Technical University of Dresden (Dresden, Germany) by Wollensak et al. ${ }^{47}$ This protocol, which is still considered as the standard CXL protocol, includes the removal of the central $9 \mathrm{~mm}$ of corneal epithelium and an application of $0.1 \%$ riboflavin for 30 minutes. After ensuring a minimal corneal thickness of $400 \mu \mathrm{m}$ and a completely riboflavin-impregnated corneal stroma, the procedure continues by applying riboflavin for another 30 minutes combined with an energy fluence of $5.4 \mathrm{~J} / \mathrm{cm}^{2}\left(3 \mathrm{~mW} / \mathrm{cm}^{2}\right)$ of UV-A light delivered in the corneal tissue. ${ }^{47}$

Since the introduction of the Dresden protocol, numerous long-term studies have been demonstrated its high safety and efficacy. ${ }^{49,50}$ After establishing the original protocol, current studies concentrate on further improving the methodology. These approaches mainly include performing the CXL without removing the epithelium ("epi-on"-CXL), accelerating the treatment (A-CXL), and/ or to customize it by focusing the CXL on the cone area (C-CXL). ${ }^{51}$

The main reason for focusing on potential transepithelial or "epi-on-CXL"- protocols is the hope to decrease the post-CXL pain-level, infection-rate, and wound healingrelated problems after the epithelial removal without compromising the effectiveness of the epi-off-CXL. The main obstacle in epi-on-CXL is the high blocking property of the epithelium for UV-A and for large hydrophilic molecules like riboflavin. Studies on epi-off-CXL could demonstrate that the desired stiffening effect is achieved by a sufficient treatment penetration of 250 to $300 \mu \mathrm{m}$ of stromal depth. ${ }^{52}$ Despite achieving the desired perioperative benefits of the epi-on-strategy, up to date, even with enhanced riboflavin solutions or iontophoresisassisted protocols, no epi-on-CXL procedure reaches this amount of cross-linked volume, potentially indicating a decreased (long-term) efficacy. ${ }^{53}$ However, new protocols including different UV-A applications (pulsed/different wave lights) and the supplementary use of oxygen and/ 
or ozone to increase the intrastromal radical-creation are currently under review. ${ }^{54}$ The accelerated strategies (A-CXL) focus on the potential benefit of shortening the treatment time of 60 minutes of the original protocol by increasing the irradiation intensity. Although current research could demonstrate the safety and short-term efficacy of A-CXL, ${ }^{54}$ a current review article analyzing different irradiation settings refers to several studies, which consistently demonstrate the appearance of more superficial demarcation lines when shortening the exposure time (and simultaneously increasing the energydose), suggesting a reduced (volume-based) stiffening of the cornea. ${ }^{55}$

The C-CXL represents a different approach: Using an eye-tracker-guided adjustable UV-A source and preoperative topography/tomography data, customized energy profiles can be projected onto the cornea. ${ }^{56,57}$ These protocols are based on the assumption that there is no need to strengthen the entire cornea, but only the biomechanical weakened area to avoid a progressive destabilization in KC eyes. Thereby, the weaker parts have to be treated with a higher intensity to ensure the necessary strengthening of the tissue. ${ }^{58}$ Analyzing C-CXL compared with the standard Dresden protocol, two different study groups report a significantly stronger decrease of Kmax and a significantly higher regularization of the corneal surface with equally deep demarcation lines in the cone-area one year after the treatment. ${ }^{56,57}$ Encouraged by their results, Seiler et al ${ }^{57}$ discussed the possibilities of further customization of the treatment to potentially improve the individual refractive outcome. However, up to date, only 1-year follow-up data are available comparing C-CXL and the Dresden-protocol-CXL. ${ }^{54,56,57}$ Long-term analyses have to proof the equality of both protocols especially in terms of long-term biomechanical stabilization. To sum up, despite all the upcoming, highly interesting modifications, presently, the original Dresden-"epi-off"-CXL remains the gold standard in increasing the biomechanical properties in progressive KC eyes, especially when the young patient presents with a good uncorrected- or spectacle/CL-corrected visual acuity. ${ }^{48}$

\section{Intracorneal Ring Segments}

Intracorneal ring segments (ICRSs) are small implants of polymethylmethacrylate plastic available in numerous arc-lengths, thickness, and designs. They were initially designed to correct low myopia in normal eyes ${ }^{59}$ but since first used by Colin et $\mathrm{al}^{60}$ for $\mathrm{KC}$, their most common indication is the treatment of ectatic corneal disease.

They function as spacers elements between the collagen fibers of the cornea and therefore, induce an arc shortening effect of the corneal geometry that induces a flattening at the central area of the cornea and a regularization of the asymmetry of the tissue. ${ }^{61}$ These changes lead to a reduction in the keratometric readings and an improvement in the refraction and vision of KC patients.

Indications for ICRS implantation are a corrected distance visual acuity below 0.9 in the decimal scale, inability to use CLs, and the absence of a visually significant central leucoma.

Two main types of ICRSs are being used nowadays for the treatment of ectatic corneal disease, different in profile and diameter of implantation: INTACS (Addition technologies) with a hexagonal cross section, 6.77 inner diameter, and a single arc length of $150^{\circ}$, and Kerarings (Mediphacos) with a triangular cross section, 5 or $6 \mathrm{~mm}$ inner diameter, and different arc lengths from $90^{\circ}$ up to $340^{\circ}$. Thickness of the intracorneal rings ranges from 0.25 to $0.35 \mathrm{~mm}$ with INTACS and 0.15 to 0.35 with Kerarings.

There are several implantation nomograms, but the most used are the ones developed by the main manufacturers of ICRS in order to decide the number, arc length, thickness, and position of the segments in the cornea. Frequently, authors advocate implanting the corneal ring guided by the steepest meridian, ${ }^{62}$ but others prefer to guide implantation by the comatic axis. ${ }^{63}$ The ICRSs are inserted into stromal tunnels at a desired depth of between 60 and $80 \%$ either manually using a handheld corkscrew blade or automatically using a femtosecond laser.

Most of the studies on ICRS implantation for the treatment of $\mathrm{KC}$ have reported an improvement in the uncorrected and corrected visual acuity, a reduction in the spherical equivalent and cylinder, a flattening of keratometric readings between 3 and $5 \mathrm{D}$, and a positive impact in optical quality by reducing anterior corneal higher order aberrations, such as coma and coma like. ${ }^{61}$ In a multicentric study it was shown that the worse the visual impairment at the time of the surgery the better the results, while patients with fairly good visual acuity were more prone to lose lines of visions after the procedure. ${ }^{64}$ Regression and further progression is found 5 years after ICRS treatment and is often a cause for ICRS explantation. ${ }^{65,66}$

Implanting ICRS in keratoconic patients is a safe surgical procedure specially when using femtosecond laser to create the tunnels. During surgery, segment decentration, asymmetric positioning of the ICRS, or inadequate depth of the tunnels are the main complications. ${ }^{67}$ After the surgery there has been described segment migration and extrusion, corneal neovascularization, corneal melting, infectious keratitis, 
and hydrops. ${ }^{66}$ Often it is found a white deposit around the segment inside the tunnels composed of fatty acids and that produces no visual impairment. ${ }^{68}$

\section{Corneal IntraStromal Implantation System/MyoRing}

Corneal IntraStromal Implantation System (CISIS) including MyoRing is a newer technology characterized by creating a lamellar pocket and insertion of a complete full-ring implant (MyoRing) into this pocket via a lamellar incision. Both the surgical technique and the implant itself is different from ICRS. This technology was developed to overcome the shortcomings of ICRS in order to avoid ICRS-related complications, such as extrusions, to improve visual rehabilitation and to achieve long-term stability and to stop progression; CISIS/MyoRing is described in detail.

\section{Keratoplasty}

The traditional choice of treatment for advanced KC is a corneal graft. ${ }^{69}$ However, there is no general consensus for the definition of advanced disease. Most corneal specialists would agree that corneal transplant would be advisable when the optical correction with spectacles is insufficient, when continued CL wear is intolerable, or when corrected visual acuity has reduced to unacceptable levels. ${ }^{70}$ The percentage of KC patients requiring a corneal transplantation is estimated to be 12 to $20 \%{ }^{71}$

Traditionally penetrating keratoplasty (PK) has been the primary choice. Recently, deep anterior lamellar keratoplasty (DALK) techniques are becoming more popular as it reduces intrasurgical risks from open-sky surgery, and almost disappears the risk of immunological rejection or late progressive endothelial cell loss. ${ }^{69}$ The reported visual and refractive outcomes in PK and DALK seem to be equivalent, although a vision of 20/20 might be more likely after PK. ${ }^{72-75}$ Taking the disadvantages of DALK like a given surgical inexperience, certain issues concerning the residual stromal thickness and DM folds into consideration, the advantages mentioned before compensate for this difference. ${ }^{69}$ One major issue when performing DALK remains the intraoperative perforation of the DM, which may occur in up to $50 \%$ of the eyes. ${ }^{70}$ Risk factors therefore, are corneal scarring near the DM, corneal ectasias with a remaining corneal thickness of less than $250 \mu \mathrm{m}$ as well as an inexperienced surgeon. ${ }^{76}$ The presence of a double anterior chamber secondary to breaks in DM, interface haze, interface infectious keratitis, and interface vascularization has also been described in DALK sugery. ${ }^{70}$
In conclusion, elective PK should be reserved for those cases when the DM and the endothelium appear splitted (e.g., after a corneal hydrops) or when there is scarring involving the DM. It may also be a better option in cases of surgeon inexperience or clinics with a low volume of keratoplasty surgery. ${ }^{69}$

\section{CORNEAL INTRASTROMAL IMPLANTATION SYSTEM/MYORING}

\section{Surgical Procedure}

As described elsewhere, ${ }^{77}$ an intrastromal pocket is created by means of the PocketMaker Ultrakeratome at a depth of $300 \mu \mathrm{m}$ with a diameter of $9.0 \mathrm{~mm}$. A lamellar incision of $5.0 \mathrm{~mm}$ width is created whereas the pocket stays closed along the remaining circumference in order to preserve the biomechanics of the tissue. The criterion for safe use of the PocketMaker Ultrakeratome in order to avoid corneal perforation is a corneal thickness of at least $350 \mu \mathrm{m}$ at the thinnest point. Via the lamellar incision the MyoRing is implanted into the intrastromal pocket. The MyoRing is produced of modified polymethylmethacrylate.

The selection of the right MyoRing dimension (diameter and thickness) is performed by processing the eye exam data securely uploaded online to DIOPTEX. The calculation is based on a sophisticated corneal model proven and optimized by more than 5,000 treatments. In order to achieve the best possible result in any given case the surgeons should follow the related treatment guidelines. $^{78}$

The PocketMaker Ultrakeratome is in particular designed for the optimal performance of the CISIS/ MyoRing surgical procedure. The procedure can alternatively also be performed via a femtosecond laser instead of the PocketMaker Ultrakeratome. ${ }^{79}$ However, since the quality of the cutting-interface created by means of the femtosecond laser at $300 \mu \mathrm{m}$ depth is much lower than that of the PocketMaker Ultrakeratome the visual results and the therapeutic index are limited. The PocketMaker uses a vibrating diamond blade guided on micron level with an extremely precise cutting edge so that the cut interface in the tissue is perfectly smooth. In contrast the corneal pocket created by the femtosecond laser is essentially not smoothly cut but disrupted. This results in a comparably rough interface and less effectiveness in utilizing the appropriate MyoRing dimension for establishing an improved corneal shape when using the femtosecond laser. ${ }^{80,81}$

\section{Visual Rehabilitation}

Inserting the MyoRing into the corneal pocket forces the cornea central to the MyoRing into a more regular shape 
and because of the perfect interface between the anterior and the posterior lamellae created by the PocketMaker Ultrakeratome; both lamellae glide against each other into a new and stable biomechanical equilibrium. ${ }^{80}$ The new corneal shape after treatment is relatively stable 3 months after initial surgery. ${ }^{82}$ Depending on the preoperative visual acuity the improvement of uncorrected visual acuity can range from 1 to 20 lines. In order to be able to achieve the best possible result in any given case it is important to calculate the individually right implant dimension via the company's online tools and to follow the optimized treatment guidelines. The procedure eliminates the negative impact of the positioning of the implant on the results as seen in ICRS treatment since it allows positioning according to the real postoperative optical axis. ${ }^{83}$ The long-term results of CISIS/MyoRing treatment of KC show that there is virtually no regression (progression) but even further improvement of the visual result over time. ${ }^{78,84}$ The explanation is that due to the natural rigidity of the tissue the corneal shape centrally to the MyoRing is much more regular after surgery but may not be perfectly regular immediately after the surgery. However, intraocular pressure permanently pushes the corneal tissue against the MyoRing, which results in a continuous "ironing" of the remaining corneal irregularities into a more regular shape day by day and year by year within the closed boundaries of the MyoRing. ${ }^{78,84}$ According to the mathematical discipline called topology the excellent visual rehabilitation in $\mathrm{KC}$ treatment by means of CISIS is therefore, the result of both, the regular and uniform cross section along the circumference of the MyoRing and the fact that the MyoRing is a

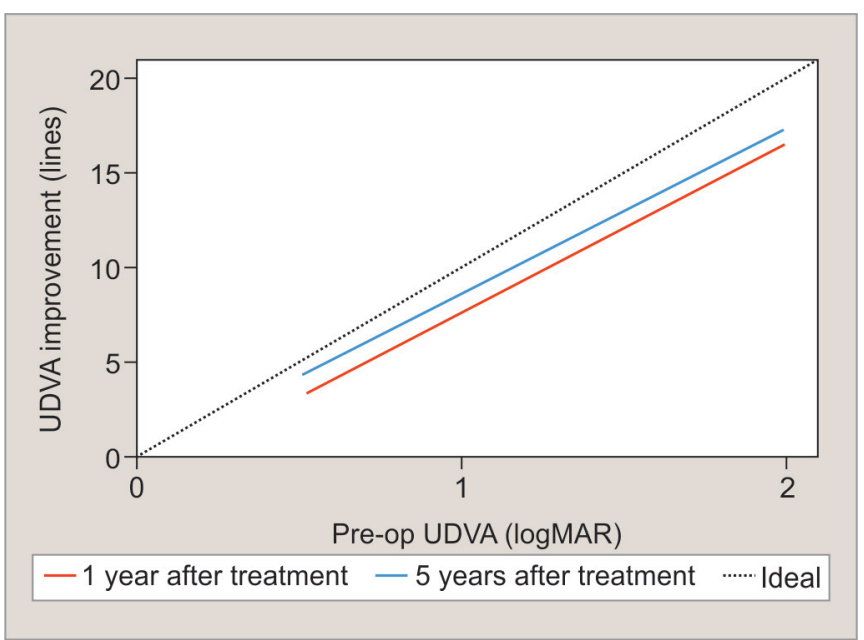

Graph 1: Linear regression analysis of line improvement vs preoperative uncorrected distance visual acuity 1 year $(r e d)$ and 5 years (b/ue) after treatment. The dotted line represents the potential for maximum improvement to $1.0(20 / 20)$ visual acuity. The shift of the regression line toward the dotted line demonstrates further significant improvement of visual acuity. Data taken from Daxer et al $^{78}$ complete and continuous ring. ${ }^{77,83}$ This effect is shown in Graphs 1 and 2 by shifting the linear regression line of the visual improvement toward the ideal line of visual correction of 20/20 vision over time. It is also important to note that CISIS/MyoRing is equal effective in both, central and non-central KC. ${ }^{85}$

\section{Biomechanics of CISIS/MyoRing}

As explained in chapter "Ultrastructure and Biomechanics of the cornea" the collagen fibrils of the cornea turn into a circular direction when approaching the corneal periphery and the limbus. This results in an increased modulus of elasticity (Young's modulus) and stabilizes the cornea there. The cornea can therefore, be considered as mounted on the limbus. ${ }^{32}$ When inserting the MyoRing into the cornea at $300 \mu \mathrm{m}$ depth the intraocular pressure pushes the convex front-surface of the MyoRing into the anterior stromal lamellae and compresses the stroma right in front of the MyoRing from 300 to $70 \mu \mathrm{m}$. That process anchors the implanted MyoRing in the stroma. The MyoRing is a continuous and complete ring, which defines a plane of mechanical support in the direction of the laminar forces inside the cornea. The MyoRing therefore, acts as a second limbus inside the cornea and therefore, stabilizes the cornea accordingly. ${ }^{33}$ In contrast to cross-linking that stabilizes the cornea by changing the elastic properties of the cornea at an ultrastructural level, the MyoRing acts as an additional support, which is able to take up the forces acting inside the cornea. It can be considered in analogy of a cast around a broken leg or a ceiling beam, which supports the ceiling in a house. This is only possible because the MyoRing is a complete

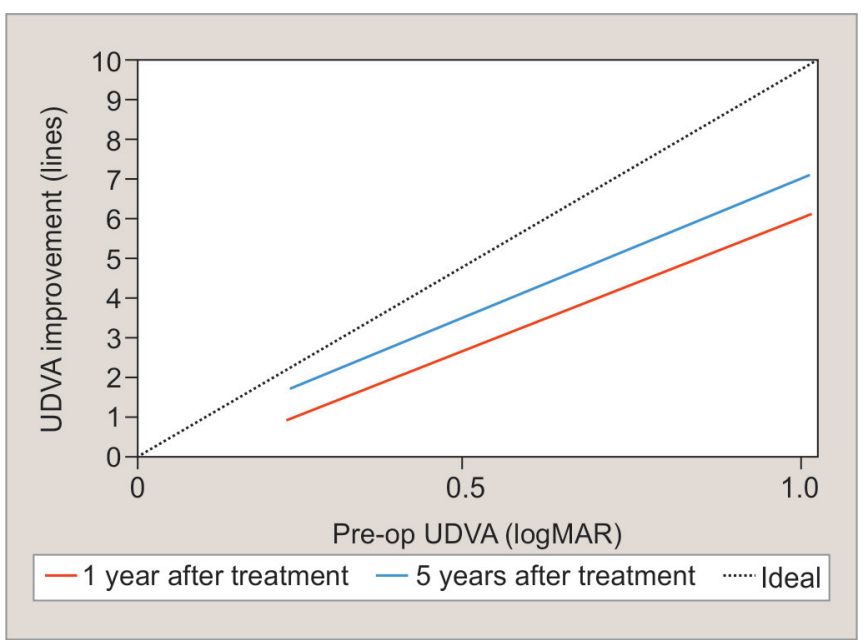

Graph 2: Linear regression analysis of line improvement vs preoperative corrected distance visual acuity 1 year $(r e d)$ and 5 years (blue) after treatment. The dotted line represents the potential for maximum improvement to $1.0(20 / 20)$ visual acuity. The shift of the regression line toward the dotted line demonstrates further significant improvement of visual acuity. The data were from Daxer et $\mathrm{al}^{78}$ 


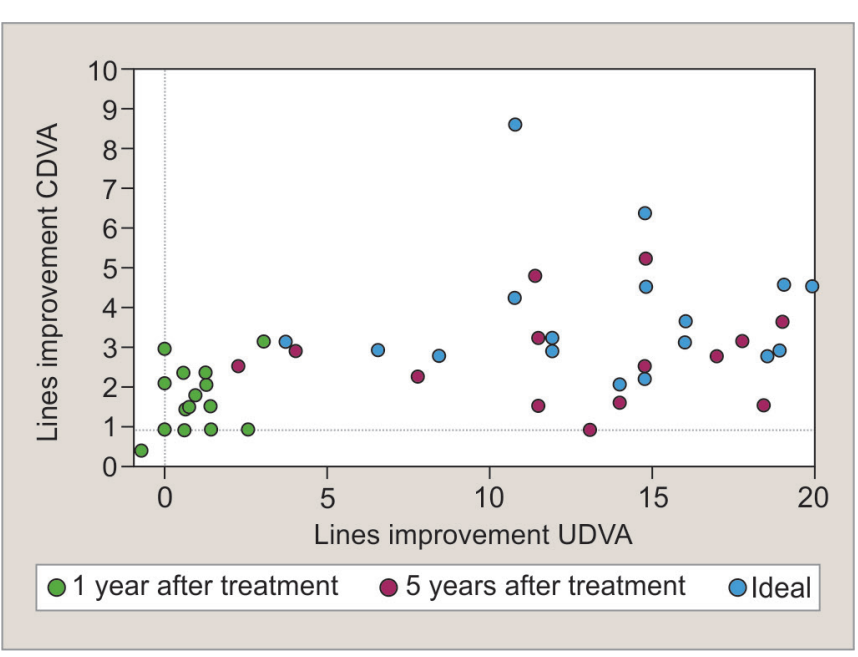

Graph 3: Individual long-term visual stability shown as line improvement of corrected distance visual acuity vs line improvement of uncorrected distance visual acuity) between preoperative and 1 year (red), between preoperative and 5 years (blue), and between 1 and 5 years (green) after treatment. The data show that there is still improvement in most of the cases between 1 and 5 years after treatment. The data were taken from Prangl-Grötzl et al ${ }^{84}$

and continuous ring implant. It is of course not possible if a ring implant is incomplete, such as ICRS. This quality is important to explain the long-term stability (Graphs 2 and 3) of CISIS/MyoRing treatment of KC. ${ }^{78,84}$

Essentially it is possible to combine CISIS/MyoRing and cross-linking to achieve both visual rehabilitation and stop of progression. ${ }^{86,87}$ However, the MyoRing alone can strengthen the cornea by a factor of three without performing cross-linking (Graph 4). The combination of CISIS/MyoRing and cross-linking should therefore, be reserved to the very unlikely event only of progression after CISIS/MyoRing treatment. ${ }^{78,87}$

\section{Complications and Side-effects}

Side-effects after treatment include postoperative glare and halos, over- or undercorrection, and decentration of the MyoRing.

Over- or undercorrection as well as decentration of the MyoRing will usually result in an exchange of the implanted MyoRing. According to the published literat ure, ${ }^{78,82,84,88}$ this occurs in between 10 and $24 \%$ of cases.

Glare and halos are reported by the patients especially in the early postoperative phase. Prangl-Grötzl et $\mathrm{al}^{84}$ report that about $37 \%$ of patients complain of disturbing glare after 3 to 5 months postoperatively. However, the number of patients reporting halos is reduced to merely $20 \%$ after 1 year, while this side-effect was not evaluated as being disturbing significantly.

Possible complications after the implantation of a MyoRing are very rare in the hand of a trained surgeon and include perforation of the cornea, extrusion of the MyoRing, or infections. There is no published data on

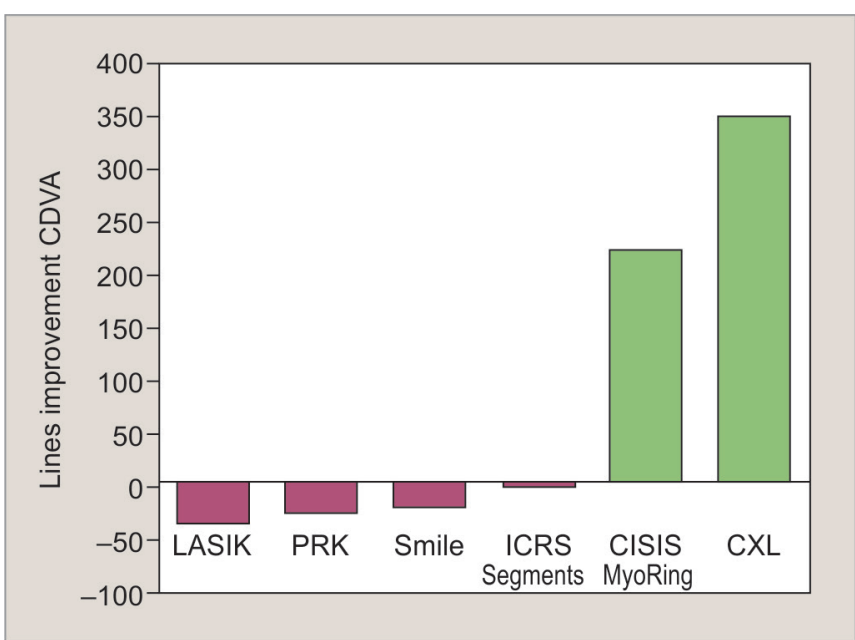

Graph 4: Change in corneal strength induced by different kinds of treatments. Data taken from Daxer ${ }^{33}$

severe complication. One group reported the performing of keratoplasty ( 2 out of 48 eyes) or the removal of the MyoRing ( 1 out of 48 eyes) after the initial implantation of a MyoRing due to patient dissatisfaction. ${ }^{88}$ It is therefore, important to inform patients who suffer from very advanced $\mathrm{KC}$, which is beyond the treatment range of MyoRing (average central $\mathrm{K}>60$ ) that the postoperative visual acuity after treatment may be not satisfactory and that keratoplasty may be the right choice in such a case. Complication rates according to the experience of the authors of this review estimate the incidence of extrusion $(<1 \%)$, corneal infection $(<0.1 \%)$, or corneal perforation $(<0.1 \%)$ to be very low.

\section{CONCLUSION}

As conclusion of this information we propose an optimized treatment scheme and a related grading for $\mathrm{KC}$.

Grade I: Uncorrected visual acuity $\geq 0.8(20 / 25)$ and no progression

Action: No treatment

Grade II: Uncorrected visual acuity $\geq 0.8(20 / 25)$ and progression

\section{Action: CXL*}

Grade III: Uncorrected visual acuity $<0.8(20 / 25)$ but corrected visual acuity $\geq 0.8(20 / 25)$ and no progression

Action: CISIS/MyoRing** or glasses or CL

Grade IV: Uncorrected visual acuity $<0.8(20 / 25)$ but corrected visual acuity $\geq 0.8(20 / 25)$ and progression and corneal thickness $\geq 400 \mu \mathrm{m}$

Action: CISIS/MyoRing or [(glasses or CL) plus crosslinking]

Grade V: Uncorrected visual acuity $<0.8(20 / 25)$ but corrected visual acuity $\geq 0.8(20 / 25)$ and progression and corneal thickness $<400 \mu \mathrm{m}$

Action: CISIS/MyoRing** 
Grade VI: Corrected visual acuity $<0.8(20 / 25)$ and no progression

Action: CISIS/MyoRing** or CL

Grade VII: Corrected visual acuity $<0.8$ and progression and corneal thickness $\geq 400 \mu \mathrm{m}$

Action: CISIS/MyoRing** or (cross-linking plus CL)

Grade VIII: Corrected visual acuity $<0.8$ and progression and corneal thickness $<400 \mu \mathrm{m}$

Action: CISIS/MyoRing**

Grade IX: Corneal thickness $<350 \mu \mathrm{m}$ and CL tolerated

Action: CL

Grade X: Corneal thickness $<350 \mu \mathrm{m}$ and CL intolerance

Action: Keratoplasty

*if corneal thickness $\geq 400 \mu \mathrm{m}$ : a criterion that is expected to be always fulfilled at that stage of the disease.

$* *$ if corneal thickness is $\geq 350 \mu \mathrm{m}$.

\section{REFERENCES}

1. Rabinowitz YS. Keratoconus. Surv Ophthalmol 1998 Jan-Feb;42(4):297-319.

2. Rabinowitz YS, Rasheed K. KISA\% index: a quantitative videokeratography algorithm embodying minimal topographic criteria for diagnosing keratoconus. J Cataract Refract Surg 1999 Oct;25(10):1327-1335.

3. Spoerl E, Huhle M, Seiler T. Induction of cross-links in corneal tissue. Exp Eye Res 1998 Jan;66(1):97-103.

4. Cavas-Martinez F, De la Cruz Sanchez E, Nieto Martinez J, Fernández-Pacheco DG. Corneal topography in keratoconus: state of the art. Eye Vis (Lond) 2016 Feb;3:5.

5. Steinberg J, Aubke-Schultz S, Frings A, Hülle J, Druchkiv V, Richard G, Katz T, Linke SJ. Correlation of the KISA\% index and Scheimpflug tomography in 'normal', 'subclinical', 'keratoconus-suspect' and 'clinically manifest' keratoconus eyes. Acta Ophthalmol 2015 May;93(3):e199-e207.

6. Rio-Cristobal A, Martin R. Corneal assessment technologies: current status. Surv Ophthalmol 2014 Nov-Dec;59(6):599-614.

7. LuzA,Lopes B,HallahanKM,ValbonB,RamosI,Faria-CorreiaF, Schor P, Dupps WJ Jr, Ambrósio R Jr. Enhanced combined tomography and biomechanics data for distinguishing forme fruste keratoconus. J Refract Surg 2016 Jul;32(7):479-494.

8. Li Y, Chamberlain W, Tan O, Brass R, Weiss JL, Huang D. Subclinical keratoconus detection by pattern analysis of corneal and epithelial thickness maps with optical coherence tomography. J Cataract Refract Surg 2016 Feb;42(2):284-295.

9. Saad A, Gatinel D. Evaluation of total and corneal wavefront high order aberrations for the detection of forme fruste keratoconus. Invest Ophthalmol Vis Sci 2012 May;53(6): 2978-2992.

10. Bühren J, Schaffeler T, Kohnen T. Validation of metrics for the detection of subclinical keratoconus in a new patient collective. J Cataract Refract Surg 2014 Feb;40(2):259-268.

11. Vellara HR, Patel DV. Biomechanical properties of the keratoconic cornea: a review. Clin Exp Optom 2015 Jan;98(1):31-38.

12. Luz A, Lopes B, Hallahan KM, Valbon B, Fontes B, Schor P, Dupps WJ Jr, Ambrósio R Jr. Discriminant value of custom ocular response analyzer waveform derivatives in forme fruste keratoconus. Am J Ophthalmol 2016 Apr;164:14-21.
13. Vinciguerra R, Ambrosio R Jr, Elsheikh A, Roberts CJ, Lopes B, Morenghi E, Azzolini C, Vinciguerra P. Detection of keratoconus with a new biomechanical index. J Refract Surg 2016 Dec;32(12):803-810.

14. Woodward MA, Blachley TS, Stein JD. The association between sociodemographic factors, common systemic diseases, and keratoconus: an analysis of a nationwide heath care claims database. Ophthalmology 2016 Mar;123(3):457. e2-465.e2.

15. Kennedy RH, Bourne WM, Dyer JA. A 48-year clinical and epidemiologic study of keratoconus. Am J Ophthalmol 1986 Mar;101(3):267-273.

16. Godefrooij DA, de Wit GA, Uiterwaal CS, Imhof SM, Wisse RP. Age-specific incidence and prevalence of keratoconus: a nationwide registration study. Am J Ophthalmol 2017 Mar;175:169-172.

17. Nielsen K, Hjortdal J, Aagaard Nohr E, Ehlers N. Incidence and prevalence of keratoconus in Denmark. Acta Ophthalmol Scand 2007 Dec;85(8):890-892.

18. Gorskova EN, Sevost'ianov EN. Epidemiology of keratoconus in the Urals. Vestn Oftalmol 1998 Jul-Aug;114(4):38-40.

19. Jonas JB, Nangia V, Matin A, Kulkarni M, Bhojwani K. Prevalence and associations of keratoconus in rural Maharashtra in central India: the central India eye and medical study. Am J Ophthalmol 2009 Nov;148(5):760-765.

20. Shneor E, Millodot M, Gordon-Shaag A, Essa M, Anton M, Barabara R, Barbara A. Prevalence of keratoconus among young Arab students in Israel. Int J Kerat Ect Cor Dis 2014 Jan-Apr;3(1):9-14.

21. Georgiou T, Funnell CL, Cassels-Brown A, O'Conor R. Influence of ethnic origin on the incidence of keratoconus and associated atopic disease in Asians and white patients. Eye (Lond) 2004 Apr;18(4):379-383.

22. Pearson AR, Soneji B, Sarvananthan N, Sandford-Smith JH. Does ethnic origin influence the incidence or severity of keratoconus? Eye (Lond) 2000 Aug;14 (Pt 4):625-628.

23. Seiler T, Huhle S, Spoerl E, Kunath H. Manifest diabetes and keratoconus: a retrospective case-control study. Graefes Arch Clin Exp Ophthalmol 2000 Oct;238(10):822-825.

24. El Sayed MO, Ali NH. Incidence and indices of keratoconus in patients presenting for LASIK in Egypt. Int J Kerat Ect Cor Dis 2017 Jan-Jun;6(1):17-22.

25. Aydin Kurna S, Altun A, Gencaga T, Akkaya S, Sengor T. Vision related quality of life in patients with keratoconus. J Ophthalmol 2014 Apr;2014:694542.

26. Rebenitsch RL, Kymes SM, Walline JJ, Gordon MO. The lifetime economic burden of keratoconus: a decision analysis using a Markov model. Am J Ophthalmol 2011 May;151(5):768. e2-773.e2.

27. Yu T, Rajendran V, Griffith M, Forrester JV, Kuffova L. Highrisk corneal allografts: a therapeutic challenge. World J Transplant 2016 Mar;6(1):10-27.

28. Daxer A, Misof K, Grabner B, Ettl A, Fratzl P. Collagen fibrils in the human corneal stroma: structure and aging. Invest Ophthalmol Vis Sci 1998 Mar;39(3):644-648.

29. Fratzl P, Daxer A. Structural transformation of collagen fibrils in corneal stroma during drying: an x-ray scattering study. Biophys J 1993 Apr;64(4):1210-1214.

30. Daxer A, Fratzl P. Collagen fibril orientation in the human corneal stroma and its implication to keratoconus. Invest Ophthalmol Vis Sci 1997 Jan;38(1):121-128. 
31. Newton RH, Meek KM. Circumcorneal annulus of collagen fibrils in the human limbus. Invest Ophthalmol Vis Sci 1998 Jun;39(7):1125-1134.

32. Daxer A. Biomechanics of the cornea. Int J Kerat Ect Cor Dis 2014 May-Aug;3(2):57-62.

33. Daxer A. Biomechanics of corneal ring implants. Cornea 2015 Nov;34(11):1493-1498.

34. Fick AE. A contact-lens. 1888 (translation). Arch Ophthalmol 1988 Oct;106(10):1373-1377.

35. Kymes SM, Walline JJ, Zadnik K, Gordon MO; Collaborative Longitudinal Evaluation of Keratoconus study group. Quality of life in keratoconus. Am J Ophthalmol 2004 Oct;138(4): 527-535.

36. Downie LE, Lindsay RG. Contact lens management of keratoconus. Clin Exp Optom 2015 Jul;98(4):299-311.

37. Bilgin LK, Yilmaz S, Araz B, Yüksel SB, Sezen T. 30 years of contact lens prescribing for keratoconic patients in Turkey. Cont Lens Anterior Eye 2009 Feb;32(1):16-21

38. Barnett M, Mannis MJ. Contact lenses in the management of keratoconus. Cornea 2011 Dec;30(12):1510-1516.

39. Ghosh S, Mutalib HA, Sharanjeet-Kaur, Ghoshal R, Retnasabapathy S. Effects of contact lens wearing on keratoconus: a confocal microscopy observation. Int J Ophthalmol 2017 Feb;10(2):228-234.

40. Bitirgen G, Ozkagnici A, Malik RA, Oltulu R. Evaluation of contact lens-induced changes in keratoconic corneas using in vivo confocal microscopy. Invest Ophthalmol Vis Sci 2013 Aug;54(8):5385-5391.

41. Hovakimyan M, Guthoff R, Reichard M, Wree A, Stachs O. In vivo confocal laser-scanning microscopy to characterize wound repair in rabbit corneas after collagen cross-linking. Clin Exp Ophthalmol 2011 Apr;39(9):899-909.

42. Marino GK, Santhiago MR, Santhanam A, Torricelli AA, Wilson SE. Regeneration of defective epithelial basement membrane and restoration of corneal transparency after photorefractive keratectomy. J Refract Surg 2017 May;33(5): 337-346.

43. Jinabhai A, Neil Charman W, O'Donnell C, Radhakrishnan H. Optical quality for keratoconic eyes with conventional RGP lens and simulated, customised contact lens corrections: a comparison. Ophthalmic Physiol Opt 2012 May;32(3):200-212.

44. Jinabhai A, O'Donnell C, Tromans C, Radhakrishnan H. Optical quality and visual performance with customised soft contact lenses for keratoconus. Ophthalmic Physiol Opt 2014 Sep;34(5):528-539.

45. Negishi K, Kumanomido T, Utsumi Y, Tsubota K. Effect of higher-order aberrations on visual function in keratoconic eyes with a rigid gas permeable contact lens. Am J Ophthalmol 2007 Dec;144(6):924-929.

46. Lee JL, Kim MK. Clinical performance and fitting characteristics with a multicurve lens for keratoconus. Eye Contact Lens 2004 Jan;30(1):20-24.

47. Wollensak G, Spoerl E, Seiler T. Riboflavin/ultraviolet-ainduced collagen crosslinking for the treatment of keratoconus. Am J Ophthalmol 2003 May;135(5):620-627.

48. Galvis V, Tello A, Ortiz AI, Escaf LC. Patient selection for corneal collagen cross-linking: an updated review. Clin Ophthalmol 2017 Apr;11:657-668.

49. Galvis V, Tello A, Ortiz AI. Corneal collagen crosslinking with riboflavin and ultraviolet for keratoconus: long-term follow-up. J Cataract Refract Surg 2015 Jun;41(6):1336-1337.

50. Raiskup F, Theuring A, Pillunat LE, Spoerl E. Corneal collagen crosslinking with riboflavin and ultraviolet-a light in progressive keratoconus: ten-year results. J Cataract Refract Surg 2015 Jan;41(1):41-46.

51. Mastropasqua L. Collagen cross-linking: when and how? A review of the state of the art of the technique and new perspectives. Eye Vis (Lond) 2015 Nov;2:19.

52. Schumacher S, Mrochen M, Wernli J, Bueeler M, Seiler T. Optimization model for UV-riboflavin corneal cross-linking. Invest Ophthalmol Vis Sci 2012 Feb;53(2):762-769.

53. Mazzotta C, Hafezi F, Kymionis G, Caragiuli S, Jacob S, Traversi C, Barabino S, Randleman JB. In vivo confocal microscopy after corneal collagen crosslinking. Ocul Surf 2015 Oct;13(4):298-314.

54. O'Brart DP. Corneal collagen crosslinking for corneal ectasias: a review. Eur J Ophthalmol 2017 May;27(3):253-269.

55. Medeiros CS, Giacomin NT, Bueno RL, Ghanem RC, Moraes HV Jr, Santhiago MR. Accelerated corneal collagen crosslinking: technique, efficacy, safety, and applications. J Cataract Refract Surg 2016 Dec;42(12):1826-1835.

56. Cassagne M, Pierne K, Galiacy SD, Asfaux-Marfaing MP, Fournié P, Malecaze F. Customized topography-guided corneal collagen cross-linking for keratoconus. J Refract Surg 2017 May;33(5):290-297.

57. Seiler TG, Fischinger I, Koller T, Zapp D, Frueh BE, Seiler T. Customized corneal cross-linking: one-year results. Am J Ophthalmol 2016 Jun;166:14-21.

58. Roberts CJ, Dupps WJ Jr. Biomechanics of corneal ectasia and biomechanical treatments. J Cataract Refract Surg 2014 Jun;40(6):991-998.

59. Burris TE. Intrastromal corneal ring technology: results and indications. Curr Opin Ophthalmol 1998 Aug;9(4):9-14.

60. Colin J, Cochener B, Savary G, Malet F. Correcting keratoconus with intracorneal rings. J Cataract Refract Surg 2000 Aug;26(8):1117-1122.

61. Vega-Estrada A, Alio JL. The use of intracorneal ring segments in keratoconus. Eye Vis 2016 Mar;3(1):8.

62. Alió JL, Shabayek MH, Artola A. Intracorneal ring segments for keratoconus correction: long-term follow-up. J Cataract Refract Surg 2006 Jun;32(6):978-985

63. Alfonso JF, Lisa C, Merayo-Lloves J, Fernández-Vega Cueto L, Montés-Micó R. Intrastromal corneal ring segment implantation in paracentral keratoconus with coincident topographic and coma axis. J Cataract Refract Surg 2012 Sep;38(9):1576-1582.

64. Vega-Estrada A, Alio JL, Brenner LF, Javaloy J, Plaza Puche AB, Barraquer RI, Teus MA, Murta J, Henriques J, UcedaMontanes A. Outcome analysis of intracorneal ring segments for the treatment of keratoconus based on visual, refractive, and aberrometric impairment. Am J Ophthalmol 2013 Mar;155(3): 575.e1-584.e1.

65. Alio JL, Vega-Estrada A, Esperanza S, Barraquer RI, Teus MA, Murta J. Intrastromal corneal ring segments: how successful is the surgical treatment of keratoconus? Middle East Afr J Ophthalmol 2014 Jan-Mar;21(1):3-9.

66. Chhadva P, Yesilirmak N, Cabot F, Yoo SH. Intrastromal corneal ring segment explantation in patients with keratoconus: causes, technique, and outcomes. J Refract Surg 2015 Jun; 31(6):362-397.

67. Miranda D, Sartori M, Francesconi C, Allemann N, Ferrara P, Campos M. Ferrara intrastromal corneal ring segments for severe keratoconus. J Refract Surg 2003 Nov-Dec;19(6):645-653.

68. Ruckhofer J, Twa MD, Schanzlin DJ. Clinical characteristics of lamellar channel deposits after implantation of intacs. J Cataract Refract Surg 2000 Oct;26(10):1473-1479. 
69. Arnalich-Montiel F, Alió del Barrio JL, Alió JL. Corneal surgery in keratoconus: which type, which technique, which outcomes? Eye Vis (Lond) 2016 Jan;3(1):2.

70. Parker JS, van Dijk K, Melles GRJ. Treatment options for advanced keratoconus: a review. Surv Ophthalmol 2015 Sep-Oct;60(5):459-480.

71. Jhanji V, Sharma N, Vajpayee RB. Management of keratoconus: current scenario. Br J Ophthalmol 2011 Aug;95(8):1044-1050.

72. MacIntyre R, Chow S-P, Chan E, Poon A. Long-term outcomes of deep anterior lamellar keratoplasty versus penetrating keratoplasty in Australian keratoconus patients. Cornea 2014 Jan;33(1):6-9.

73. Han DC, Mehta JS, Por YM, Htoon HM, Tan DT. Comparison of outcomes of lamellar keratoplasty and penetrating keratoplasty in keratoconus. Am J Ophthalmol 2009 Nov;148(5):744.e1-751.e1.

74. Fontana L, Parente G, Sincich A, Tassinari G. Influence of graft-host interface on the quality of vision after deep anterior lamellar keratoplasty in patients with keratoconus. Cornea 2011 May;30(5):497-502.

75. Jones MN, Armitage WJ, Ayliffe W, Larkin DF, Kaye SB, NHSBT Ocular Tissue Advisory Group and Contributing Ophthalmologists (OTAG Audit Study 5). Penetrating and deep anterior lamellar keratoplasty for keratoconus: a comparison of graft outcomes in the United Kingdom. Invest Ophthalmol Vis Sci 2009 Dec;50(12):5625-5629.

76. Baradaran-Rafii A, Eslani M, Sadoughi M-M, Esfandiari H, Karimian F. Anwar versus Melles deep anterior lamellar keratoplasty for keratoconus: a prospective randomized clinical trial. Ophthalmology 2013 Feb;120(2):252-259.

77. Daxer A. Corneal intrastromal implantation surgery for the treatment of moderate and high myopia. J Cataract Refract Surg 2008 Feb;34(2):194-198
78. Daxer A, Ettl A, Hörantner R. Long-term results of MyoRing treatment of keratoconus. J Optom 2017 Apr-Jun;10(2): 123-129.

79. Alio JL, Pinero DP, Daxer A. Clinical outcome after complete ring implantation in corneal ectasia using the femtosecond laser technology: a pilot study. Ophthalmology 2011 Jul;118(7):1282-1290.

80. Daxer A. MyoRing treatment of keratoconus. Int J Kerat Ect Cor Dis 2015 May-Aug;4(2):76-83.

81. Hosny M, El-Mayah E, Sidky MK, Anis M. Femtosecond laserassisted implantation of complete versus incomplete rings for keratoconus treatment. Clin Ophthalmol 2015 Jan;9:121-127.

82. Daxer $\mathrm{A}, \mathrm{Mahmoud} \mathrm{H}$, Venkateswaran RS. Intracorneal continuous ring implantation for keratoconus: one-year follow-up. J Cataract Refract Surg 2010 Aug;36(8):1296-1302.

83. Daxer A. Adjustable intracorneal ring in a lamellar pocket for keratoconus. J Refract Surg 2010 Mar;26(3):217-221.

84. Prangl-Grötzl A, Ettl A, Hörantner R, Daxer A. Individual long-term visual stability after MyoRing treatment of keratoconus. Int J Kerat Ect Cor Dis 2016 May-Aug;5(2):53-56.

85. Daxer A. MyoRing for central and noncentral keratoconus. Int J Kerat Ect Cor Dis 2012 May-Aug;1(2):117-119.

86. Studeny P, Krizova D, Stranak Z. Clinical outcome after complete intracorneal ring implantation and corneal collagen cross-linking in an intrastromal pocket in one session for keratoconus. J Ophthalmol 2014 Sep;2014:Article ID 568128.

87. Daxer A, Mahmoud HA, Venkateswaran RS. Corneal crosslinking and visual rehabilitation in keratoconus in one session without epithelial debridement: new technique. Cornea 2010 Oct;29(10):1176-1179.

88. Janani L, Jadidi K,MosaviSA,NejatF,NaderiM,NourijelyaniK. MyoRing implantation in keratoconic patients: 3 years follow-up data. J Ophthalmic Vis Res 2016 Jan-Mar;11(1):26-31. 